\title{
Complete regression of ductal carcinoma in situ following chemotherapy and trastuzumab: should we rethink management?
}

\begin{abstract}
Background: Ductal carcinoma in situ (DCIS) includes comedo-DCIS, which consists of high-grade DCIS associated with dilated ducts filled with intraluminal comedonecrosis. Current consensus opinion is that prognosis is excellent for all forms of DCIS and that no form of DCIS responds to chemotherapy. However, herein we describe a patient who had complete resolution of comedo-DCIS with the combination of paclitaxel, carboplatin and trastuzumab and results of a retrospective analysis that challenge current opinion.
\end{abstract}

Materials and methods: The cancer registry of Hoag Hospital in Newport Beach, California, was used to indentify subset cohorts of patients with DCIS and local-stage invasive breast cancer. Survival curves were compared by the log-rank test.

Results: There were 7,373 breast cancer patients diagnosed during 1983-2007 including 205 classified as comedo-DCIS, 1006 as non-comedo-DCIS, 46 as locally invasive comedocarcinoma and 3905 as other types of locally invasive breast cancer. Ninety-eight percent of the comedocarcinoma diagnoses were made prior to 1997 . During 1991-2000 comedo-DCIS accounted for 134/505 (26.5\%) of all DCIS compared to $61 / 565(10.8 \%)$ during 2001-2007 $(\mathrm{p}<0.0001)$. Patients with comedoDCIS had a worse overall survival than other DCIS patients $(\mathrm{p}<0.040)$, but survival was similar for comedo-DCIS and invasive comedocarcinoma $(\mathrm{p}=0.62)$.

Conclusion: Neoadjuvant chemotherapy plus trastuzumab can completely eradicate Her2-positive comedo-DCIS. Survival for comedo-DCIS is worse than for noncomedo DCIS and similar to invasive comedocarcinoma. Because of changes in synoptic reporting and current terminology in pathology reports, comedocarcinoma is no longer being documented and the diagnosis of comedo-DCIS has decreased. Additional studies are warranted to determine whether women with large breast lesions that show only comedo-DCIS on biopsy, should be considered candidates for neoadjuvant therapy to facilitate breast-conserving surgery.

Keywords: comedo-DCIS, comedocarcinoma, Her2-positive DCIS, neoadjuvant, chemotherapy, trastuzumab
Volume 2 Issue 5 - 2015

\author{
Robert O Dillman, ${ }^{1,2,3}$ Louis A \\ Vandermolen, ${ }^{2,3}$ F Scott Heinemann, ${ }^{1,4}$ \\ Stephanie E McClure' \\ 'Caladrius Biosciences, Hoag Hospital, USA \\ ${ }^{2}$ Hoag Family Cancer Institute, USA \\ ${ }^{3}$ Departments of Medical Oncology, Hoag Hospital, USA \\ ${ }^{4}$ Departments of Medical Pathology, Hoag Hospital, USA
}

Correspondence: Robert O Dillman, Departments of Medical Oncology, Vice President of Oncology, Caladrius Biosciences Inc., I830I Von Karman Avenue, Suite I30, USA, Tel 949-725-

1750, Email robert.dillman55@gmail.co;

rdillman@caladrius.com

Received: December I, 2014 | Published: August 14, 2015
Abbreviations: DCIS, ductal carcinoma in situ; ER, estrogen receptor; PR, progesterone receptor; Her2, human epidermal growth factor 2; IHC, immuno histochemistry; FISH, fluorescent in situ hybridization; AJCC, the american joint committee on cancer; DCISM, DCIS with microinvasion; MRI, magnetic resonance imaging; PET/CT, photon emission tomography with computerized tomography; AUC, area under the curve; LCIS, lobular carcinoma in situ; ICD-O, international classification of diseases for oncology; SEER, surveillance epidemiologic end-result; HIPAA, health insurance portability and accountability act

\section{Introduction}

The term ductal carcinoma in situ(DCIS) encompasses the spectrum of non-invasive intraductal cellular proliferations in the breast and includes comedo-DCIS, which refers to certain high-grade DCIS lesions associated with ductal dilatation and intraluminal necrosis that extrudes like the material in a comedone. ${ }^{1-5}$ Comedo-DCIS typically is associated with mutations that are also detected in high-grade invasive breast cancer in association with a high proliferative rate and a high risk of distant metastases. ${ }^{6-8}$ These include absence of estrogen receptor (ER) and progesterone receptor (PR) expression, mutations of the $\mathrm{p} 53$ suppressor gene and phenotypic over-expression of human epidermal growth factor 2 (Her2) proteins as detected by immuno histochemistry (IHC), and/or amplification of the Her2 gene defined by increased gene copies detected by fluorescent in situ hybridization (FISH). Comedo-DCIS is associated with a greater risk of local recurrence compared to other types of DCIS, but is not believed to be associated with a worse survival. ${ }^{5,9,10}$

High-grade comedo-DCIS is often found in association with invasive cancers ${ }^{11}$ that are negative for $\mathrm{ER}$ and $\mathrm{PR}$ but positive for Her2 while "triple-negative" invasive breast cancers are less often associated with high-grade DCIS. ${ }^{11,12}$ Compared to other DCIS histologies, comedo-DCIS is associated with a higher rate of microinvasion especially in larger lesions ${ }^{13,14}$ and is also more often associated with axillary lymph node metastases than other forms of DCIS. ${ }^{15}$

The American Joint Committee on Cancer (AJCC) defines DCIS with microinvasion (DCISM) as $0.1 \mathrm{~cm}$ or less of invasive cancer, 
in greatest dimension. ${ }^{16}$ Whether invasive cancer is detected in a background of extensive comedo-DCIS is important for directing patient management. Current guidelines prescribe that chemotherapy is not warranted in patients with a histological diagnosis of DCIS without documented invasive cancer. ${ }^{17}$ However, if biopsies reveal microinvasion or lymph node metastasis, then patients might be treated with neo-adjuvant chemotherapy, with or without trastuzumab depending on whether the tumor is Her2-positive. Similarly, if microinvasion and/or lymph node metastases are documented at the time of definitive surgical resection, then adjuvant systemic chemotherapy therapy is often recommended. Per cancer registry guidelines, when metastases are identified in regional lymph nodes, but only DCIS is identified in the breast tissue, then the tumor is reclassified as node-positive infiltrating ductal carcinoma rather than comedo-DCIS, because it is assumed that invasive cancer exists somewhere in the primary breast lesion.

Does comedo-DCIS respond to chemotherapy and/or to chemotherapy plus trastuzumab? Should some patients with comedoDCIS be treated as if their primary cancer does contain microinvasion, even if infiltration is not identified, especially for larger lesions? With these questions in mind, the following case and a retrospective analysis are reported.

\section{Case report}

A 57year-old White female presented with a large right breast mass. Physical examination confirmed a large hard mass in the lateral right breast, nipple retraction and a clear discharge. Digital mammograms showed bilateral heterogeneously dense breasts, but the right breast contained extensive coarse casting calcifications and a prominent right axillary node. Ultrasound (US) demonstrated hypoechoic areas and confirmed calcifications and an enlarged lymph node. Two US-guided core biopsies were positive for $0.9 \mathrm{~cm}$ of grade 3 solid DCIS with comedonecrosis and calcifications, but no invasive cancer (Figure 1). Right axillary node biopsies revealed a $0.5 \mathrm{~cm}$ metastasis, Nottingham 3/3 and Scarf-Bloom Richardson score of 9. IHC on the lymph node metastasis was negative for ER and PR, but $3+$ positive for Her 2 and $60 \%$ of the cells expressed the proliferative marker Ki-67. The comedo-DCIS in the breast biopsies had the same staining pattern, including Her-2 positivity. Immunostains for p63 and myosin heavy chain demonstrated preservation of myoepithelial cells surrounding the tumor in the breast biopsies.

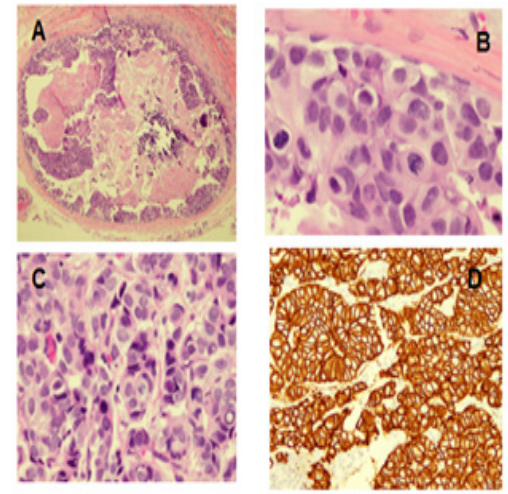

Figure I Comedo-DCIS and lymph node metastasis before neoadjuvant taxane-carboplatin-trastuzumab neoadjuvant therapy. (A) Comedonecrosis and high-grade DCIS in original breast core biopsy (hematoxylin-eosin 10x) (B) MitoticfiguresinDCISfromoriginalbreastcorebiopsy (hematoxylin-eosin80x). (C) Lymph node showing metastasis (hematoxylin-eosin I00x).

(D) Her2 expression in lymph node metastasis (immunohistochemical stain 40x).
Magnetic resonance imaging (MRI) confirmed a $9.3 \times 2.8 \times 11.0 \mathrm{~cm}$ heterogenous, segmental lesion in the lateral aspect of the right breast extending from the nipple to the axillary tail. Several abnormal appearing lymph nodes were noted. Photon emission tomography with computerized tomography (PET/CT) revealed a hypermetabolic region in the right breast and a single $1.5 \times 1.0 \mathrm{~cm}$ hypermetabolic right axillary lymph node, but no distant metastatic disease. She was staged as TX N1 M0.

The treatment plan was for six cycles of neo-adjuvant TCH (docetaxel $75 \mathrm{mg} / \mathrm{m}^{2}$, carboplatin at an area under the curve (AUC) of 6 and trastuzumab $6 \mathrm{mg} / \mathrm{kg}$ ) given every 21 days, followed by surgery and then an additional year of trastuzumab. However, because of toxicity, starting with cycle two the regimen was changed to weekly paclitaxel, $80 \mathrm{mg} / \mathrm{m}^{2}$, carboplatin at AUC 2 and trastuzumab $2 \mathrm{mg} /$ $\mathrm{kg}$. After two months the breast mass and axillary adenopathy had resolved. After four months, PET/CT showed regression of the lateral right breast mass with persistent calcifications. MRI showed resolution of the lymph node and the breast mass had decreased to $7.4 \times 2.0 \times 8.1 \mathrm{~cm}$.

A right mastectomy with axillary node dissection revealed a complete pathologic response. Not only was there no invasive tumor, there was also complete resolution of DCIS with only a $6 \mathrm{~cm}$ area of fibrosis and ducts with calcifications, fibrosis and inflamed stroma. The ductal epithelium, which was originally Her2-positive, was Her2negative in the surgical specimen and the cells lining the ducts showed smaller cells with squamous differentiation in contrast to the original biopsies that showed large and high-grade cells. A sentinel lymph node and 10 additional nodes were negative for cancer, although two nodes contained fibrosis suggesting treated tumor.

\section{Patients and methods}

Patients diagnosed with breast cancer during 1983-2007 were identified in the cancer registry of Hoag Hospital in Newport Beach, California; therefore, all patients had the potential for at least five years of follow up from diagnosis. Patients were sub-classified into pure lobular carcinoma in situ (LCIS), comedo-DCIS, non-comedo DCIS and local stage invasive breast cancer based on registry classifications. In recording histology, registrars used the terminology provided in pathology reports as prescribed in the International Classification of Diseases for Oncology (ICD-O). ${ }^{18}$ Per registry requirements, if a pathologist used the terms "comedo-DCIS," or "non-invasive comedocarcinoma," histology was coded to $8501 / 2$ ( 2 is the behavior code that signifies non-invasive) and reported as "comedocarcinoma not infiltrating," regardless of whether other types of in situ carcinoma were present. If a pathologist used the terms "comedocarcinoma" or "invasive comedocarcinoma," the histology was coded to $8501 / 3$ ( 3 is the behavior code that signifies invasion) and reported as "comedocarcinoma." Patients with regional or distant spread of disease were excluded and any patient with DCIS and lymph node metastasis was classified as TX rather than Tis. ${ }^{16}$

Reports with actuarial survival data were generated using registry software provided by Electronic Registry Systems, Inc., Cincinnati, OH. Follow-up information was available on more than $90 \%$ of living patients at five years and for more than $80 \%$ of all living patients at 10years. Analyses were limited to "analytical cases," defined as patients who were diagnosed at the institution and/or who received cancer therapy at the institution within four months of initial diagnosis. Proportions were compared using the chi-square test. Survival curves were compared using the Mantel-Haenszel log-rank test. All p-values were 2 -sided with $\mathrm{p} \leq 0.05$ considered significant. 


\section{Results}

There were 7,373 breast cancer patients diagnosed during the years 1983-2007; 6,398 with invasive cancer and 1305 with in situ cancer. Subsets of DCIS included 205 (17\%) comedo-DCIS and 1211 non-comedo-DCIS. Of those with invasive cancer, 3951 were classified as local stage disease (negative for local extension or regional lymph node or distant metastases) including 46 who were classified as comedocarcinoma (1.2\%). Table 1 lists the numbers of patients with local-stage infiltrating comedocarcinoma or comedoDCIS by the year in which they were diagnosed. The distribution of comedocarcinoma is clearly skewed with $42 / 46$ (91\%) diagnosed between the years 1990 and 1996 and only one patient diagnosed as comedocarcinoma between 1997 and 2007. In addition, there were 13 patients with lymph node metastases and two with distant disease that were classified as comedocarcinoma at diagnosis who are not included in these analyses. Fourteen of these 15 higher-stage patients were diagnosed during 1990-1996, but only one subsequently. The frequency of the diagnosis of comedo-DCIS also decreased. During the years 1991-2000 comedo-DCIS accounted for 134/505 (26.5\%) of all DCIS compared to 61/565 (10.8\%) during the years 2001-2007 $(p<0.0001)$. It is doubtful that the use of myoepithelial stains to help identify microinvasion explains such a dramatic decline in frequency of comedo-DCIS after $2000^{19}$ especially since the frequency of diagnosis of invasive comedocarcinoma declined even more.

Comedo-DCIS accounted for 205/1211(16.9\%) of all DCIS while comedocarcinoma accounted for only 46/3951(1.2\%) of all invasive cancer. This probably greatly underestimates the numbers of cases of comedo-DCIS plus locally invasive cancer because the focus of pathology reports is on the invasive cancer unless the DCIS is particularly extensive. As shown in Table 1, after 1996 the diagnosis of comedocarcinoma and the pathology descriptions that led registrars to code for comedocarcinoma virtually disappeared from the lexicon. However, even during 1990-1996, when comedo-DCIS was $25.3 \%$ of DCIS diagnoses, comedocarcinoma accounted for only $4.4 \%$ of localized invasive cancer.

Survival for comedo-DCIS was worse compared to non-comedo DCIS patients (Figure 2) with an absolute difference of five percentage points at five years. However, there was no difference in survival curves for comedo-DCIS, comedocarcinoma and non-comedo locally invasive (T1-T3 N0 M0) cancer (Figure 3). In the subset of patients with T1N0M0 disease, there was also no difference in survival for 30 comedocarcinoma patients compared to 2507 invasive non-comedo cancer patients $(\mathrm{p}=0.42)$. The median follow up of surviving patients was greater than 10 years for each cohort. Treatments administered within the first four months following a diagnosis of comedo-DCIS or comedocarcinoma are shown in Table 2. Not surprisingly, the treatments utilized differed significantly based on whether patients were classified as comedo-DCIS as opposed to comedocarcinoma $(\mathrm{p}<0.0001)$. Typically only regional therapies were used in patients with comedo-DCIS, while there was an increased use of systemic therapy in patients with comedocarcinoma.

Table I Numbers of cases of local stage invasive comedocarcinoma and as a percent of all local stage invasive breast cancers, and comedo-DCIS diagnosed each year and as a percent of all ductal carcinoma in situ (DCIS)

\begin{tabular}{|c|c|c|c|c|c|c|}
\hline $\begin{array}{l}\text { Accession } \\
\text { Year }\end{array}$ & $\begin{array}{l}\text { Number of cases } \\
\text { of local comedo } \\
\text { carcinoma }\end{array}$ & $\begin{array}{l}\text { Number of cases of } \\
\text { local non-comedo } \\
\text { carcinoma }\end{array}$ & $\begin{array}{l}\text { Percent of All local } \\
\text { invasive }\end{array}$ & $\begin{array}{l}\text { Number of cases of } \\
\text { Comedo DCIS }\end{array}$ & $\begin{array}{l}\text { Number of } \\
\text { cases of non- } \\
\text { Comedo-DCIS }\end{array}$ & $\begin{array}{l}\text { Comedo DCIS } \\
\text { percentage of all } \\
\text { DCIS }\end{array}$ \\
\hline 1983 & 0 & 69 & 0 & $I$ & 5 & 16.7 \\
\hline 1984 & I & 61 & 1.6 & 0 & 9 & 0 \\
\hline 1985 & I & 89 & I.I & 0 & 9 & 0 \\
\hline 1986 & 0 & 89 & 0 & I & 16 & 5.9 \\
\hline 1987 & 0 & 74 & 0 & I & 14 & 6.7 \\
\hline 1988 & I & 112 & 0.9 & I & 28 & 3.4 \\
\hline 1989 & 0 & 103 & 0 & 4 & 24 & 14.2 \\
\hline 1990 & 2 & 97 & 2.0 & 2 & 26 & 7.1 \\
\hline |99| & 4 & 115 & 3.4 & 11 & 18 & 37.9 \\
\hline 1992 & 14 & 117 & 10.7 & 19 & 30 & 38.8 \\
\hline 1993 & 7 & 130 & 5.1 & 10 & 36 & 21.7 \\
\hline 1994 & 10 & 147 & 6.4 & 14 & 26 & 35.0 \\
\hline 1995 & 3 & 160 & 1.8 & 10 & 43 & 18.9 \\
\hline 1996 & 2 & 180 & I.I & 7 & 36 & 16.2 \\
\hline 1997 & 0 & 153 & 0 & 11 & 43 & 20.3 \\
\hline 1998 & 0 & 179 & 0 & 13 & 49 & 21.0 \\
\hline 1999 & 0 & 203 & 0 & 23 & 43 & 34.8 \\
\hline 2000 & 0 & 196 & 0 & 16 & 47 & 25.4 \\
\hline 2001 & 0 & 227 & 0 & 7 & 81 & 8.0 \\
\hline 2002 & 0 & 220 & 0 & II & 68 & 13.9 \\
\hline 2003 & 0 & 203 & 0 & 5 & 61 & 7.6 \\
\hline 2004 & 0 & 221 & 0 & 11 & 66 & 14.3 \\
\hline 2005 & 0 & 238 & 0 & 11 & 79 & 12.2 \\
\hline 2006 & I & 243 & 0.4 & 5 & 68 & 6.8 \\
\hline 2007 & 0 & 283 & 0 & 11 & 81 & 12.0 \\
\hline Total Cases & 46 & 3905 & 1.2 & 205 & 1006 & 16.9 \\
\hline
\end{tabular}


Table 2 Distribution of therapies initiated during the first four months after a diagnosis of comedo-DCIS or comedocarcinoma (comedo DCIS plus locally invasive breast cancer)

\begin{tabular}{llll}
\hline Initial Therapy & Comedo DCIS & Comedocarcinoma & Total \\
\hline Surgery alone & $113(55 \%)$ & $23(50 \%)$ & 137 \\
Surgery+RT & $52(25 \%)$ & $7(15 \%)$ & 59 \\
Surgery+Hormone \pm RT & $35(17 \%)$ & $10(22 \%)$ & 45 \\
Surgery+Chemo \pm RT & $5(3 \%)$ & $6(13 \%)$ & $1 \mathrm{I}$ \\
Total & 205 & 46 & $25 \mathrm{I}$ \\
\hline
\end{tabular}

DCIS=Ductal Carcinoma in Situ; RT = Radiation Therapy; Chemo = Chemotherapy

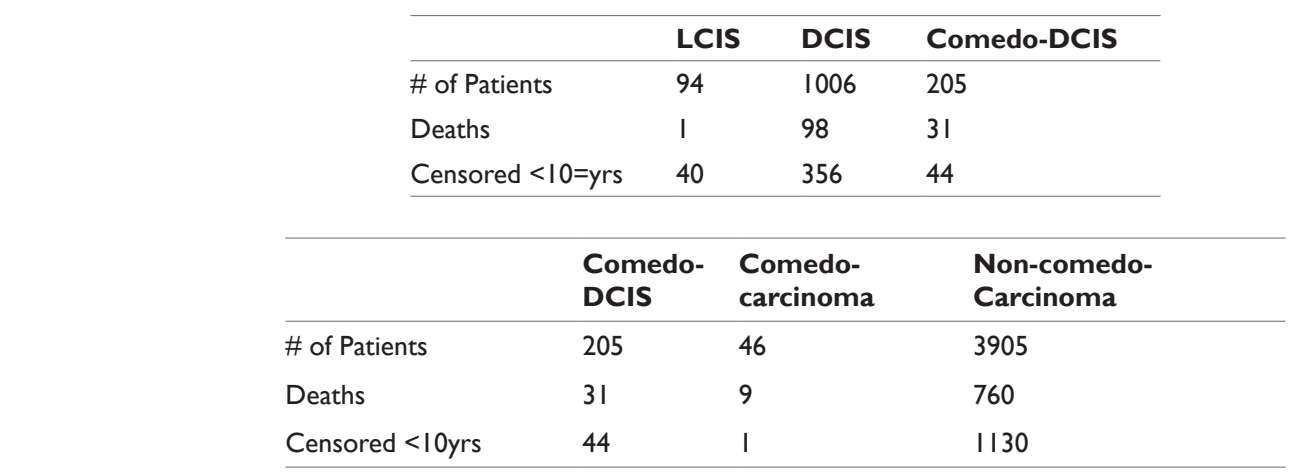

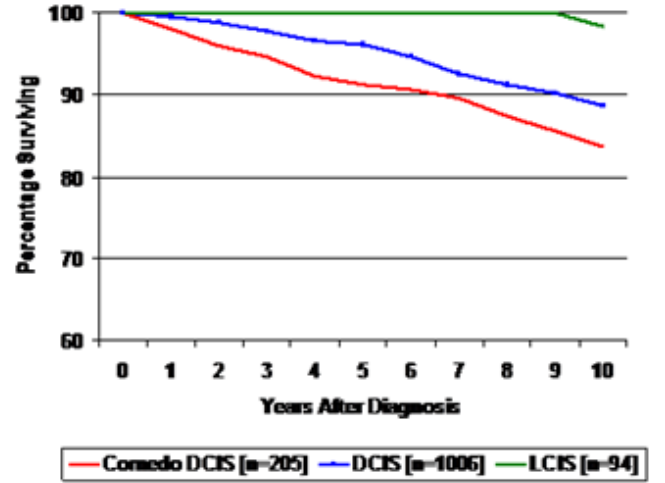

Figure 2 Overall survival for patients diagnosed with lobular carcinoma in situ (LCIS), non-comedo ductal carcinoma in situ with or without LCIS (DCIS), and comedo-DCIS for patients diagnosed during 1983-2007. Survival for DCIS was worse than LCIS $(p<0.00 I)$; survival for comedo-DCIS was worse than for non-comedo DCIS $(p<0.040)$.

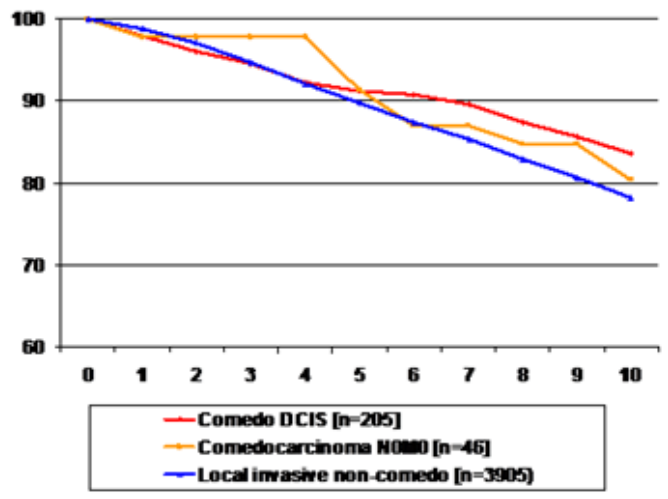

Figure 3 Overall survival for patients diagnosed during 1983-2007 with (A) comedo-DCIS $(n=205)$. (B) Locally invasive comedocarcinoma $(n=46)$. (C) locally invasive non-comedo carcinoma (3905). Log-rank tests: comedo-DCIS vs comedocarcinoma $(p=0.62)$, comedocarcinoma vs locally invasive noncomedocarcinoma $(p=0.70)$, comedo-DCIS vs locally invasive non-comedo cancer $(\mathrm{p}=0.09)$.

\section{Discussion}

Herein we report a complete pathologic remission of extensive comedo-DCIS following neoadjuvant therapy with a combination of chemotherapy plus trastuzumab and provide data suggesting that patients with comedo-DCIS have a survival that is more similar to that of comedo-DCIS plus microinvasion (comedocarcinoma), than to the survival of patients with non-comedo DCIS. These observations raise important questions regarding current consensus recommendations for the management of this subclass of DCIS. Most studies of DCIS have focused on disease recurrence rather than overall survival and comedo-DCIS is consistently associated with a higher rate of local recurrence in the ipsilateral breast. ${ }^{5,13,20}$ We identified only two reports that compared all-cause mortality for patients with comedo and noncomedo DCIS. In a study of 227patients diagnosed with DCIS between 1979 and 1990, there was no difference in survival for comedo versus non-comedo DCIS histologies..$^{21}$ However, the numbers of patients in the study were relatively small and the median follow up was less than five years. In a smaller study of 172 women with a median follow up of seven years, there also was no difference in survival for patients with comedo versus non-comedo DCIS. ${ }^{22}$

DCIS is not felt to be responsive to systemic therapy, ${ }^{23}$ but our case and another recent report ${ }^{24}$ show that comedo-DCIS can be completely eradicated with chemotherapy and trastuzumab. The two cases were similar in terms of a mammographic picture of branching calcifications, high-grade DCIS with comedo necrosis, the presence of lymph node metastasis which justified the use of neoadjuvant therapy and the use of trastuzumab because of Her2 over expression. In both cases a complete pathologic response was documented at the time of mastectomy following neoadjuvant therapy, despite the persistence of calcifications radiographically. In the other case the tumor was ER-positive and Her2-positive and the patient received doxorubicin and cyclophosphamide followed by paclitaxel and trastuzumab; ${ }^{24}$ our patient was ER-negative and received taxane and carboplatin in combination with trastuzumab. These reports demonstrate that Her2 positive comedo-DCIS can be responsive to chemotherapy plus trastuzumab, but our case demonstrates that an anthracyline is not 
necessary to achieve a complete pathologic response in such patients.

Patients with large breast lesions and no lymphadenopathy, whose biopsies show only comedo-DCIS, currently are not offered neoadjuvant therapy as a strategy to facilitate breast conservation..$^{25}$ The observation that complete pathologic responses can be achieved in patients with HER2-positive comedo-DCIS suggests that breast conservation might be achieved in such patients with neoadjuvant therapy and is worthy of further study. It is unclear whether such a result could be achieved with neoadjuvant trastuzumab alone.

Patients with ER-negative, Her-2 positive, high-grade invasive breast cancer who receive neoadjuvant anthracycline-based therapy and achieve a complete response, including absence of DCIS in mastectomy specimens, have an excellent prognosis. ${ }^{26}$ In that series complete pathologic responses were noted in $33 \%$ of 298 patients with high-grade, ER-negative, Her2-positive cancer who received trastuzumab and chemotherapy and in $28 \%$ of 239 such patients who did not receive trastuzumab. ${ }^{26}$ However, the report did not provide information regarding the presence or absence of DCIS in biopsies before neoadjuvant therapy was given; so, it is unclear how often DCIS was eliminated by these therapies. In patients with high-grade, ER-positive, Her2-positive cancer, complete pathologic responses were observed in $22 \%$ of 356 patients who received chemotherapy plus trastuzumab, but in only $12 \%$ who did not receive trastuzumab. ${ }^{26}$

There is no general agreement among pathologists regarding the definition of comedo DCIS, ${ }^{9,27}$ although it has been suggested that identification of only one dilated duct filled with necrotic cellular material may be sufficient for this designation. ${ }^{1}$ Some have considered the term comedocarcinoma synonymous with comedo-DCIS, but for more than 35years this term has been used to classify comedoDCIS associated with invasive cancer. ${ }^{18}$ Microinvasive cancer can be difficult to detect; therefore, the myoepithelial stains myosin and p63 are often applied to samples of comedo-DCIS in an effort to detect breakdown of the luminal membrane to help identify occult microinvasion. ${ }^{19}$ Tumor size $\leq 1 \mathrm{~cm}$ in greatest diameter was associated with a very low rate of lymph node metastases in NSABP B-21, but only 10/638 patients had moderate to marked comedo necrosis. ${ }^{28}$ Because patients with comedo-DCIS and lymph node metastases are classified as invasive rather than in situ cancer, we could not determine how often comedo DCIS was reclassified to a higher stage because of metastases.

In the modern era DCIS usually is detected by mammography rather than palpation of a breast mass. Radiographically comedoDCIS is often associated with linear and branching calcifications that outline the ductal lumens in anatomic segments of the breast, as opposed to the focal clustered calcifications associated with low grade DCIS. ${ }^{29,30}$ In one series this pattern of casting microcalcifications was noted in only 52/714 (7\%) of women with primary invasive breast cancer that was less than $1.5 \mathrm{~cm}$ in diameter. ${ }^{31}$ The authors noted that presence of casting microcalcifications was associated with a more than three-fold higher rate of lymph node metastases, poor histologic grade and death. We did not find a difference in survival for patients with comedocarcinoma compared to patients with localized noncomedo cancer, even in patients with T1N0M0 disease.

Strengths of this study are the large numbers of patients, the longterm follow up with very few patients lost to follow up and the use of all cause mortality as the key endpoint. To our knowledge, this is the largest institutional report focusing on the long-term survival of patients with DCIS and comedo-DCIS. Limitations of this study are its retrospective nature, the limited information documented in registry-based data and the variation in the diagnoses of comedoDCIS and comedocarcinoma over time. It is noteworth that in the national Surveillance Epidemiologic End-Result (SEER) data base, the incidence of DCIS began increasing dramatically about 1984, but comedo-DCIS did not increase in incidence until 1990. ${ }^{31}$ However, DCIS continued to increase after 1994 while comedo-DCIS appeared to plateau and begin to decline after 1995. We suspect this is an artifact of the tumor registry data reported to SEER, because pathologists have become more restrictive in their use of the term comedonecrosis in their descriptions of DCIS. It is likely that a comprehensive review of pathology samples would lead to a much greater number of cases being classified in the comedo categories per traditional definitions. In the mid-1990s several investigators emphasized that nuclear grade was a more important prognostic factor than comedonecrosis architecture. In one classification system, DCIS began to be characterized as: (1) high-grade, (2) non-high-grade with necrosis, or (3) non-highgrade without necrosis, based on the associated probabilities of local recurrence for each of these subsets. ${ }^{24}$ Other systems focused on the grade and extent of the DCIS. At present synoptic pathology reports are supposed to note whether or not necrosis is present, but many local pathologists ceased using the term comedonecrosis in their reports of comedo-DCIS with invasive components; so the diagnosis of invasive comedocarcinoma has virtually disappeared. Another possible explanation is that the increased use of mammographic screening resulted in detection and treatment of DCIS that would only have become comedo-DCIS after additional mutations, but this seems unlikely.

\section{Acknowledgements}

This work was funded by Hoag Hospital and the Hoag Hospital Foundation.

\section{Ethical standards}

For this retrospective review of cancer registry data, a waiver of consent under the Common Rule for the protection of human subjects and a waiver of authorization under the Health Insurance Portability and Accountability Act (HIPAA) were obtained from the Western Institutional Review Board, Olympia, Washington.

\section{Conflict of interest}

The author declares no conflict of interest.

\section{References}

1. Fonseca R, Hartmann LC, Petersen IA, et al. Ductal carcinoma in situ of the breast. Ann Intern Med. 1997;127(11):1013-1022.

2. Burstein HJ, Polyak K, Wong JS, et al. Ductal carcinoma in situ of the breast. N Engl J Med. 2004;350(14):1430-1441.

3. Leonard GD, Swain SM. Ductal carcinoma in situ, complexities and challenges. J Natl Cancer Inst. 2004;96(12):906-920.

4. Kuerer HM, Albarracin CT, Yang WT, et al. Ductal carcinoma in situ: state of the science and roadmap to advance the field. $J$ Clin Oncol. 2008;27(2):279-288.

5. Virnig BA, Shamliyan T, Tuttle TM, et al. Diagnosis and management of ductal carcinoma in situ (DCIS). Evidence Report/Technology Assessment No. 185 (Provided by AHRQ); 2009. 
6. van de Vijver MJ, Peterse JL, Mooi WJ, et al. Neu-protein overexpression in breast cancer. Association with comedo-type ductal carcinoma in situ and limited prognostic value in stage II breast cancer. $N$ Engl J Med. 1988;319(19):1239-1245.

7. Leal CB, Schmitt FC, Bento MJ, et al. Ductal carcinoma in situ of the breast: histological categorization and its relationship to ploidy and immunohistochemnical expression of hormone receptors, p53, and c-erb2 protein. Cancer. 1995;75(8):2123-2131.

8. Allred DC. Ductal carcinoma in situ:terminology, classification, and natural history. J Natl Cancer Inst Monogr. 2010;2010(41):134-138.

9. Silverstein MJ. Ductal carcinoma in situ of the breast:controversial issues. Oncologist. 1998;3(2):94-103.

10. Bellamy CO, McDonald C, Salter DM, et al. Noninvasive ductal carcinoma of the breast: the relevance of histologic categorization. Human Pathol. 1993;24(1):16-23.

11. Pervez S, Khan H. Infiltrating ductal carcinoma breast with central necrosis closely mimicking ductal carcinoma in situ (comedo type):a case series. J Med Case Reports. 2007;1:83.

12. Bryan BB, Schnitt SJ, Collins LC. Ductal carcinoma in situ with basallike phenotype: a possible precursor to invasive basal-like breast cancer. Mod Pathol. 2006;19(5):617-621.

13. Lagios MD, Margolin FR, Westdahl PR, et al. Mammographically detected duct carcinoma in situ. Frequency of local recurrence following tylectomy and prognostic effect of nuclear grade on local recurrence. Cancer. 1989;63(4):618-624.

14. Yang M, Moriya T, Oguma M, et al. Microinvasive ductal carcinoma (T1mic) of the breast. The clinicopathological profile and immunohistochemical features of 28 cases. Pathol Int. 2003;53(7):422-428.

15. Wasserberg N, Morgenstern S, Schachter J, et al. Risk factors for lymph node metastases in breast ductal carcinoma in situ with minimal invasive component. Arch Surg. 2002;137(11):1249-1252.

16. Edge SB, Byrd DR, Compton CC, et al. AJCC Cancer Staging Manual. 7th ed. New York, USA: Springer; 2010. p. 347-376.

17. NCCN Clinical Practice Guidelines in Oncology. Breast Cancer. 2013;3:1-176.

18. Fritz A, Percy C, Jack A, et al. International Classification of Diseases for Oncology (ICD-O). 3rd ed. Geneva, Switzerland: World Health Org; 2010
19. Prasad ML, Hyjek E, Giri DD, et al. Double immunolabeling with cytokeratin and smooth-muscle actin in confirming early invasive carcinoma of the breast. Am J Surg Pathol. 1999;23(2):176-181.

20. Bellamy CO, McDonald C, Salter DM, et al. Noninvasive ductal carcinoma of the breast: the relevance of histologic categorization. Hum Pathol. 1993;24(1):16-23.

21. Silverstein MJ, Poller DN, Waisman JR, et al. Prognostic classification of breast ductal carcinoma-in-situ. Lancet. 1995;345(8958):1154-1157.

22. Solin LJ, Kurtz J, Fourquet A, et al. Fifteen-year results of breast-conserving surgery and definitive breast irradiation for the treatment of ductal carcinoma in situ of the breast. J Clin Oncol. 1996;14(3):754-763.

23. Wu W, Kamma H, Ueno E, et al. The intraductal component of breast cancer is poorly responsive to neo-adjuvant chemotherapy. Oncology Rep. 2002;9(5):1027-1031.

24. Gunia SR, Patel MS, Mamounas EP. Pathologic complete response of HER-2 Neu-positive invasive ductal carcinoma and ductal carcinoma in situ following neoadjuvant chemotherapy plus trastuzumab:a case report and review of literature. Case Rep Surg. 2012;2012:45273.

25. Schott AF, Hayes DF. Defining the benefits of neoadjuvant chemotherapy for breast cancer. J Clin Oncol. 2012;30(15):1747-1749.

26. von Minckwitz G, Untch M, Blohmer JU, et al. Definition and impact of pathologic complete response on prognosis after neoadjuvant chemotherapy in various intrinsic breast cancer subtypes. J Clin Oncol. 2012;30(15):1796-1804

27. Consensus conference on the classification of ductal carcinoma in situ. The consensus conference committee. Cancer. 1997;80(9):1798-1802.

28. Fisher ER, Costantino JP, Leon ME, et al. Pathobiology of small invasive breast cancers without metastases (T1a/b, N0, M0). National Surgical Adjuvant Breast and Bowel Project (NSABP) protocol B-21. Cancer. 2007;110(9):1929-1936.

29. Zunzunegui RG, Chung MA, Oruwari J, et al. Casting-type calcifications with invasion and high-grade ductal carcinoma in situ:a more aggressive disease. Arch Surg. 2003;138(5):537-540.

30. Barreau B, de Mascarel I, Feuga C, et al. Mammography of ductal carcinoma in situ of the breast: review of 909 cases with radiographic-pathologic correlation. Eur J Radiol. 2005;54(1):55-61.

31. Tabar L, Tony Chen HH, Amy Yen MF, et al. Mammographic tumor features can predict long-term outcomes reliably in women with 1-14 mm invasive breast carcinoma. Cancer. 2004;101(8):1745-1759. 